\title{
Rebound of relapses after discontinuation of rituximab in a patient with MOG-IgG1 positive highly relapsing optic neuritis: a case report
}

\author{
Seok-Jin Choi ${ }^{1}$, Boram Kim², Haeng-Jin Lee ${ }^{3}$ Seong-Joon Kim³ ${ }^{3}$ Sung-Min Kim²* and Jung-Joon Sung ${ }^{2}$
}

\begin{abstract}
Background: Myelin oligodendrocyte glycoprotein immunoglobulin G1 (MOG-lgG1)-associated disease is suggested as a separate disease entity distinct from multiple sclerosis and neuromyelitis optica spectrum disorder. Nonetheless, the optimal treatment regimen for preventing relapses in MOG-lgG1-associated disease remains unclear.

Case presentation: We describe the case of a 45-year-old man with MOG-lgG1-positive highly relapsing optic neuritis who had experienced 5 attacks over 21 months and had monocular blindness despite prednisolone and azathioprine therapy. He began treatment with rituximab, which reduced the rate of relapse markedly. Following discontinuation of rituximab, however, the patient experienced two successive optic neuritis attacks 2 and 4 months after B-lymphocyte restoration.

Conclusions: Highly relapsing MOG-IgG1-associated disease can be prevented with rituximab even when the MOG-IgG1 titers are relatively stationary. Discontinuation of rituximab and restoration of B-lymphocytes may be associated with the rebound of disease activity.
\end{abstract}

Keywords: MOG-IgG1, Optic neuritis, Highly relapsing, Rituximab

\section{Background}

Myelin oligodendrocyte glycoprotein immunoglobulin G1 (MOG-IgG1)-associated disease is suggested as a separate disease entity distinct from multiple sclerosis and neuromyelitis optica spectrum disorder (NMOSD) with anti-aquaporin-4 IgG (AQP4-IgG); it has a predilection for the optic nerve rather than spinal cord, perineural enhancement extending to adjacent soft tissues on magnetic resonance imaging (MRI), and a less unfavorable prognosis than NMOSD [1]. Recent studies with a sufficient number of patients and duration of follow-up have indicated that a considerable number of patients with MOG-IgG1 have relapsing attacks in the central nervous system followed by neurological deficits [2, 3]. Nonetheless, the optimal treatment regimen for preventing

\footnotetext{
* Correspondence: sueh916@gmail.com

${ }^{2}$ Department of Neurology, Seoul National University Hospital, 101,

Daehak-Ro Jongno-Gu, Seoul 03080, Republic of Korea

Full list of author information is available at the end of the article
}

relapses in patients with MOG-IgG1-associated disease has only recently begun to be studied [4]. Here, we describe a patient with highly relapsing optic neuritis $(\mathrm{ON})$ associated with MOG-IgG1, whose ON attacks were relatively well-prevented with rituximab (RTX) treatment. However, the patient experienced rebounds of repeated ON attacks shortly after the restoration of B-cells following discontinuation of RTX.

\section{Case presentation}

A 45-year-old man presented with decreased right visual acuity (VA) accompanied by periocular pain lasting for 1 week. Ophthalmological examination revealed that the patient's right eye was only able to perceive light (bestcorrected VA, light perception/0.9 in decimals, measured using a Snellen chart) and had relative afferent pupillary defect of grade 3, diffuse disc swelling, and inferior disc hemorrhage. Neurological examination showed normal muscle strength in all extremities, no sensory deficits,

(c) The Author(s). 2018 Open Access This article is distributed under the terms of the Creative Commons Attribution 4.0 International License (http://creativecommons.org/licenses/by/4.0/), which permits unrestricted use, distribution, and 
normoactive deep tendon reflexes, and no signs of bladder or bowel dysfunction. Orbit MRI revealed T2 high signal intensities and diffuse contrast enhancement along the right anterior and posterior optic nerve, as well as perineural enhancement [1] (Fig. 1-a and b). The results of cerebrospinal fluid (CSF) analysis showed a red blood cell count of $0 / \mu \mathrm{L}$, a white blood cell count of $1 / \mu \mathrm{L}$, and a protein level of $27 \mathrm{mg} / \mathrm{dL}$. CSF oligoclonal band measured by isoelectric focusing was negative and IgG index was 0.64. The result of a serum AQP4-IgG flow cytometry assay using AQP4-M23-expressing live cells was negative [5]. Right ON was suspected, and intravenous methylprednisolone ( $1000 \mathrm{mg}$ pulse therapy) for 5 days followed by oral prednisolone $(60 \mathrm{mg}$ daily) were prescribed. The right VA of the patient was improved to 0.5 (visual Functional System score improved to 2 from 5).

The second right ON attack (0.15/1.0) occurred 4 months after the first $\mathrm{ON}$ when the prednisolone dose had been tapered to $10 \mathrm{mg}$ daily. Thus, azathioprine 50 mg twice per day was started in a remission state between the second and third ON (4 months prior to the third $\mathrm{ON})$. The average thickness of a retinal nerve fiber layer measured by spectral-domain optical coherence tomography was decreased in the right eye (right $51 \mu \mathrm{m}$ and left $105 \mu \mathrm{m}$ ) (Fig. 2-a). The third (hand movement/0.9) and fourth (finger count/1.2) right ON attacks occurred 6 and 10 months after the second ON, respectively, while the prednisolone dose was maintained at 5 $\mathrm{mg}$ daily and azathioprine was $75 \mathrm{mg}$ twice per day. Following these attacks, the patient developed left central serous chorioretinopathy $(0.15 / 0.9)$ associated with long-term steroid use. The $25 \mathrm{mg}$ dose of prednisolone was thus tapered out at this point. Nevertheless, right ON recurred 2 months later for a fifth time (hand movement/0.9) when the patient was under azathioprine treatment only. At this time, the patient developed
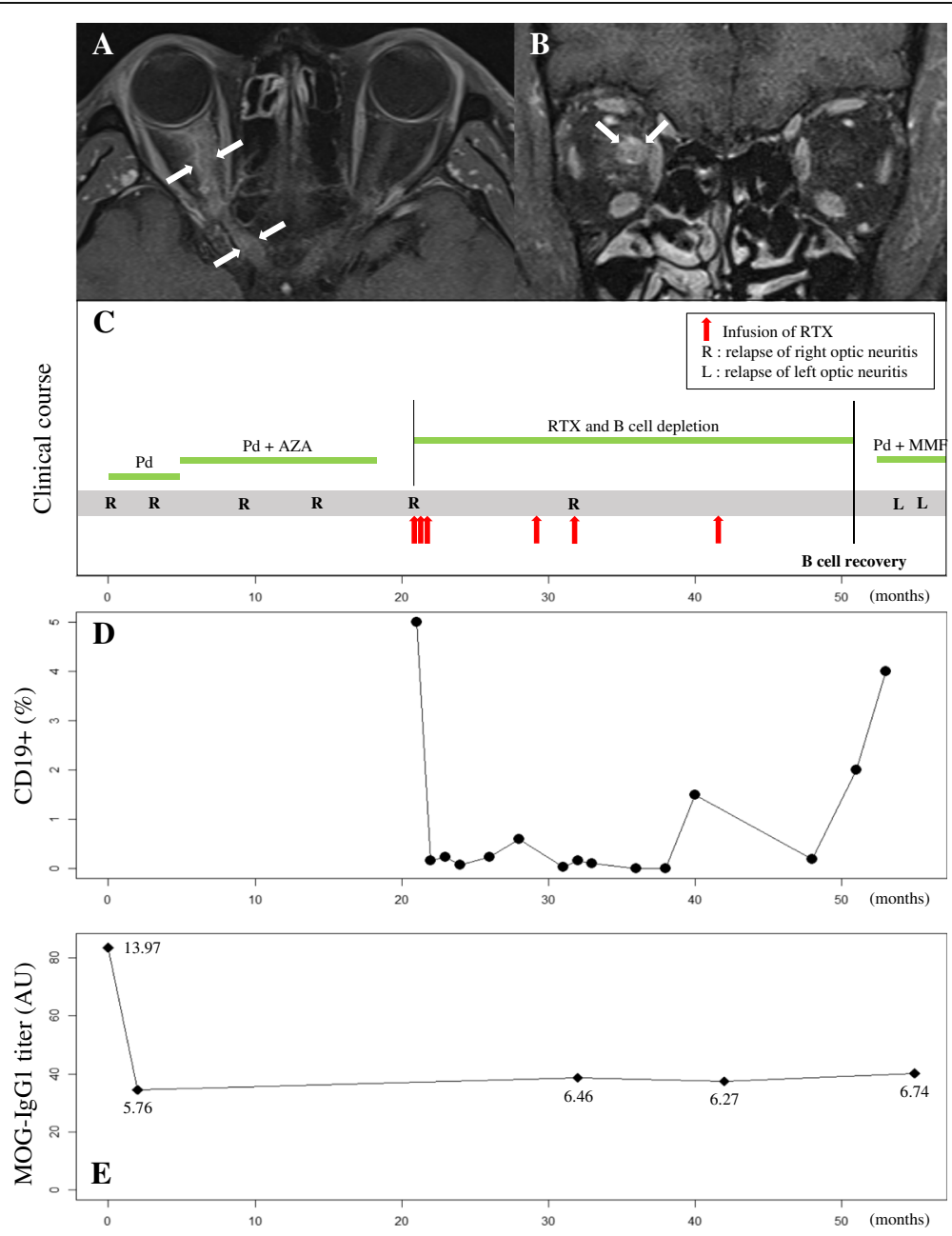

Fig. 1 (a) Axial and (b) coronal T1-weighted magnetic resonance images demonstrating diffuse gadolinium enhancement and swelling along the right anterior and posterior optic nerve. c Longitudinal clinical course of recurrent optic neuritis. d Change of CD19+ B-lymphocytes (\%) during rituximab treatment. e Change of MOG-IgG1 titers measured by a geometric mean fluorescence (G-mean) ratio of the MOG-expressing cells that bound to lgG1 using in-house flow cytometry (G-mean ratio = G-mean values of the patient's sera / G-mean values of the healthy control) 


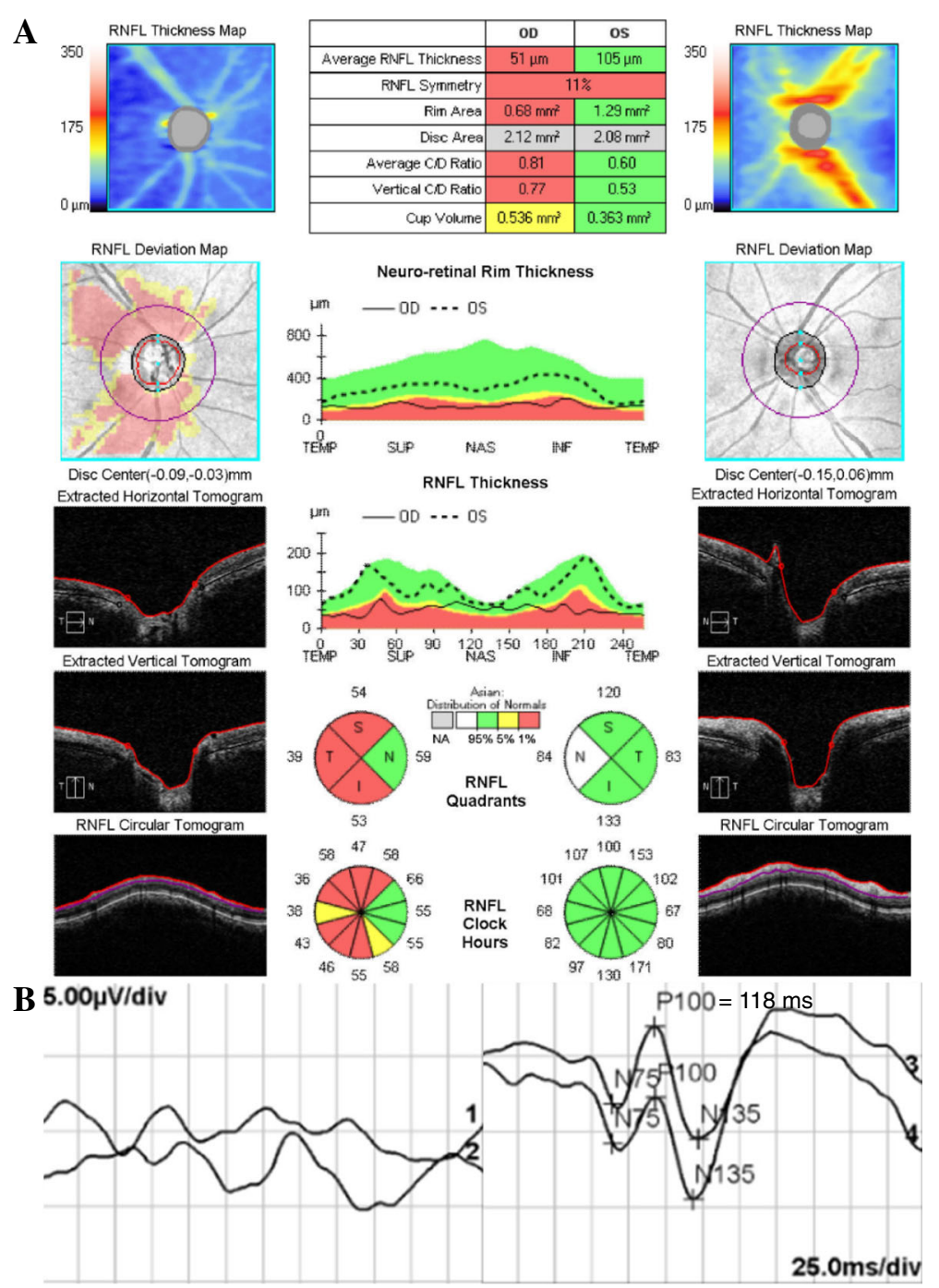

Fig. 2 a (Remission state after the second optic neuritis) the average retinal nerve fiber layer thickness measured by spectral-domain optical coherence tomography was decreased in the right eye (right $51 \mu \mathrm{m}$ and left $105 \mu \mathrm{m}$ ), with preferential thinning of the superior, temporal, and inferior quadrants. b (During fifth optic neuritis) the pattern-reversal visual evoked potential showed an abnormal waveform in the right eye with diminished amplitude. The left eye presented a relatively preserved response with prolonged P100 latency (118 ms)

monocular blindness. The pattern-reversal visual evoked potential showed an abnormal waveform in the right eye with diminished amplitude. The left eye presented a relatively preserved response with prolonged P100 latency (118 ms) (Fig. 2-b). Serum from the patient sampled at the time of the fifth ON attack was tested for MOG-IgG1 using a cell-based assay utilizing full-length human MOG (Radcliffe Hospital, Oxford, UK) [6]. The result of this test was positive.

Despite continued immunosuppressive treatment and due to the repeated ON attacks and the side effect of the steroid (chorioretinopathy), the patient was administered RTX $\left(375 \mathrm{mg} / \mathrm{m}^{2}, 3\right.$ weekly infusion for induction and 3 maintenance doses under CD19+ B-cell monitoring over 29 months). Although one mild $\mathrm{ON}$ attack (no light perception/1.2) occurred in the patient's right eye during RTX treatment, the rate of relapse decreased markedly and the patient's visual function was well-maintained. However, 32 months after the initiation of RTX treatment, we became unable to maintain RTX treatment due to insurance issues (denial for reimbursement). As a result, the treatment was switched to mycophenolate mofetil (250 mg twice per day) combined with oral prednisolone ( $5 \mathrm{mg}$ every other day). The patient's CD19+ B-lymphocyte level was restored to 2 and $4 \%$ at 9 and 11 months after the last RTX infusion, respectively. Subsequently, 2 more left $\mathrm{ON}$ attacks (hand movement/1.0 and hand movement/0.15) occurred within a one-month interval (Fig. 1-c and 1-D). The titer of MOG-IgG1 was measured by a geometric mean fluorescence (G-mean) 
ratio of the MOG-expressing cells bound to IgG1 using in-house flow cytometry. The G-mean ratio was calculated for each sera as followings: G-mean values of the patient's sera / G-mean values of the healthy control. The titer was not associated with the continuation or cessation of the RTX treatment (Fig. 1-e).

\section{Discussion and conclusions}

Here, we describe the longitudinal clinical course and treatment response to RTX therapy in a patient with MOG-IgG1-positive highly relapsing ON. We found that 1) highly relapsing MOG-IgG1-associated disease can be prevented with RTX even when the MOG-IgG1 titers are relatively preserved, and 2) discontinuation of RTX in patients with this condition can cause rebound of disease activity with restoration of B-lymphocytes.

Initial reports regarding MOG-IgG1-associated disease indicated that it typically has a monophasic and benign disease course [7]. However, recent multicenter studies have shown that a considerable proportion of patients have a relapsing course of disease, and some have significant neurological deficits [2, 3]. More recently, RTX was reported to reduce the rate of relapse in some cases of MOG-IgG1-associated disease [4]. Nevertheless, the results of studies comparing the patient's condition before vs. after the treatment should be interpreted with caution because the disease may have a naturally decreasing relapse rate in the later stages, as in NMOSD [8], and also the statistical phenomenon of regression towards the mean. In this regard, the present case, wherein we observed a restoration of B-lymphocytes and a subsequent rebound of relapses after discontinuation of RTX treatment, implies that long-term RTX maintenance therapy may be helpful in patients with highly relapsing MOG-IgG1-associated disease.

Despite initial treatment with azathioprine and prednisolone, the patient had a high relapse rate of 0.238 /year ( 5 attacks over 21 months) and subsequent unilateral visual loss in the right eye. After initiating RTX treatment, his relapse rate markedly decreased to 0.031 /year (1 attack over 32 months). However, the patient experienced $2 \mathrm{ON}$ attacks over 4 months following cessation of RTX treatment and restoration of B-lymphocytes.

In summary, the case described here illustrates that RTX can be a good treatment option for preventing relapses in MOG-IgG1-associated disease. The treatment effect was observed despite the relatively unchanged MOG-IgG1 titers during the treatment period. Finally, cessation of RTX treatment and restoration of B-lymphocytes may be associated with the rebound of disease activity. RTX may serve as an effective treatment regimen in MOG-IgG1-associated disease, especially in patients with high relapse rates.

\section{Abbreviations}

AQP4: anti-aquaporin-4; CSF: cerebrospinal fluid; lgG: immunoglobulin G; MOG: myelin oligodendrocyte glycoprotein; MRI: magnetic resonance imaging; NMOSD: neuromyelitis optica spectrum disorder; ON: optic neuritis; RTX: rituximab; VA: visual acuity

\section{Acknowledgements \\ None.}

\section{Funding}

This work was supported by grant no. HI17C0335 and HI17C0789 from the Korea Health Industry Development Institute Research fund. The funders had no role in the design of the study, interpretation of data and in writing the manuscript. They had contributed to the data collection and analysis of AQP4-IgG and MOG-IgG1 antibodies.

\section{Availability of data and materials}

All data have been presented within the manuscript and in the form of images.

\section{Authors' contributions}

S-MK conceived of the study. S-JC and S-MK analyzed and interpreted the data, and involved in drafting and revising the manuscript. BK contributed to acquisition and analysis of the data, and involved in revising the manuscript critically. J-JS contributed to interpretation of the data, and involved in revising the manuscript critically. H-JL and S-JK made substantial contributions to interpretation of ophthalmological data and also involved in revising the manuscript critically; as a result, this manuscript came to have more intellectual content on ophthalmology. All listed authors have participated sufficiently in the work to take public responsibility for appropriate portions of the content, and agreed to be accountable for all aspects of the work in ensuring that questions related to the accuracy or integrity of any part of the work are appropriately investigated and resolved.

Ethics approval and consent to participate

This study was approved by the ethics committee of Seoul National University Hospital (IRB no. 1005-023-317).

\section{Consent for publication}

Written informed consent was obtained from the patient for publication of this case report.

\section{Competing interests}

The authors declare that they have no competing interests.

\section{Publisher's Note}

Springer Nature remains neutral with regard to jurisdictional claims in published maps and institutional affiliations.

\section{Author details}

${ }^{1}$ Department of Neurology, Inha University Hospital, Incheon, Republic of Korea. ${ }^{2}$ Department of Neurology, Seoul National University Hospital, 101, Daehak-Ro Jongno-Gu, Seoul 03080, Republic of Korea. ${ }^{3}$ Department of

Ophthalmology, Seoul National University Hospital, Seoul, Republic of Korea.

Received: 6 March 2018 Accepted: 9 December 2018

Published online: 21 December 2018

References

1. Kim SM, Woodhall MR, Kim JS, Kim SJ, Park KS, Vincent A, Lee KW, Waters P. Antibodies to MOG in adults with inflammatory demyelinating disease of the CNS. Neurol Neuroimmunol Neuroinflamm. 2015;2(6):e163.

2. Jurynczyk M, Messina S, Woodhall MR, Raza N, Everett R, Roca-Fernandez A, Tackley G, Hamid S, Sheard A, Reynolds G, et al. Clinical presentation and prognosis in MOG-antibody disease: a UK study. Brain. 2017;140(12):3128-38.

3. Cobo-Calvo A, Ruiz A, Maillart E, Audoin B, Zephir H, Bourre B, Ciron J, Collongues N, Brassat D, Cotton F, et al. Clinical spectrum and prognostic value of CNS MOG autoimmunity in adults: The MOGADOR study. Neurology. 2018;90(21):e1858-e1869.

4. Ramanathan S, Mohammad S, Tantsis E, Nquyen TK, Merheb V, Fung VSC, White OB, Broadley S, Lechner-Scott J, Vucic S, et al. Clinical course, 
therapeutic responses and outcomes in relapsing MOG antibody-associated demyelination. J Neurol Neurosurg Psychiatry. 2018;89(2):127-137.

5. Yang J, Kim SM, Kim YJ, Cheon SY, Kim B, Jung KC, Park KS. Accuracy of the Fluorescence-Activated Cell Sorting Assay for the Aquaporin-4 Antibody (AQP4-Ab): Comparison with the Commercial AQP4-Ab Assay Kit. PLoS One. 2016;11(9):e0162900

6. Waters P, Woodhall M, O'Connor KC, Reindl M, Lang B, Sato DK, Jurynczyk M, Tackley G, Rocha J, Takahashi T, et al. MOG cell-based assay detects nonMS patients with inflammatory neurologic disease. Neurol Neuroimmunol Neuroinflamm. 2015;2(3):e89.

7. Kitley J, Woodhall M, Waters P, Leite MI, Devenney E, Craig J, Palace J, Vincent A. Myelin-oligodendrocyteglycoprotein antibodies in adults with a neuromyelitis optica phenotype. Neurology. 2012;79(12):1273-1277.

8. Kim SM, Park J, Kim SH, Park SY, Kim JY, Sung JJ, Park KS, Lee KW. Factors associated with the time to next attack in neuromyelitis optica: accelerated failure time models with random effects. PLoS One. 2013;8(12):e82325.

Ready to submit your research? Choose BMC and benefit from:

- fast, convenient online submission

- thorough peer review by experienced researchers in your field

- rapid publication on acceptance

- support for research data, including large and complex data types

- gold Open Access which fosters wider collaboration and increased citations

- maximum visibility for your research: over $100 \mathrm{M}$ website views per year

At BMC, research is always in progress.

Learn more biomedcentral.com/submissions 